\title{
Induction of Human Interleukin-1 by a Product of Staphylococcus aureus Associated with Toxic Shock Syndrome
}

\author{
Takashi Ikejima, Charles A. Dinarello, D. Michael Gill, \\ and Sheldon $M$. Wolff \\ Department of Medicine, Division of Experimental Medicine, and \\ Department of Molecular Biology and Microbiology, Tufts \\ University School of Medicine, Boston, Massachusetts 02111
}

bstract. Certain strains of Staphylococcus aureus associated with toxic shock syndrome elaborate material that induces human blood monocytes to secrete interleukin-1 (IL-1). IL-1 was detected both by its ability to cause fever in rabbits using the leukocytic pyrogen (LP) assay and by its mitogenic activity towards thymocytes in the so-called lymphocyte-activating factor (LAF) assay. Anti-human IL-1 prevents the manifestation of both activities. Filtrates of control strains of $S$. aureus manifest neither activity. Thus, culture filtrates derived from toxic shock syndrome (TSS)-associated strains cause biphasic fever in rabbits when injected intravenously. The fever lasts several hours. Plasma taken at the peak of the fever and injected into a second set of rabbits produces a brief monophasic fever typical of LP. Further, human monocytes release LP when incubated with TSS filtrates in vitro. The monocyte products also stimulate the proliferation of mouse thymocytes in the presence of phytohemagglutinin in a manner characteristic of LAF. A bacterial filtrate is much less effective without an intermediate incubation with monocytes. The stimulation of monocyte IL-1 production is easily quantified, provides a simple method of assaying the TSS toxin, and since it involves human cells, is directly relevant to the human disease. The assay was used to monitor the purification of TSS toxin. Only $0.1 \mathrm{ng} / \mathrm{ml}$ of the purified material is required to induce monocyte IL-1 production. It is thus more

Part of this work was presented at the 75th Annual Meeting of the American Society for Clinical Investigation, Washington, DC, 1983.

Dr. Dinarello is the recipient of a Research Career Development Award from the National Institute of Allergy and Infectious Diseases. Address all correspondence to Dr. Dinarello.

Received for publication 12 August 1983 and in revised form 19 January 1984.

J. Clin. Invest.

(c) The American Society for Clinical Investigation, Inc.

0021-9738/84/05/1312/09 \$1.00

Volume 73, May 1984, 1312-1320 potent than endotoxin. In contrast to endotoxin, its effect is not blocked by polymyxin $B$.

We conclude that in TSS the sudden fever and probably other components of the acute phase response may be attributed to a massive release of IL-1.

\section{Introduction}

Toxic shock syndrome (TSS) ${ }^{1}$ (1) has been attributed to certain strains of Staphylococcus aureus that colonize the vagina or other areas. Some of the signs and symptoms of TSS, which include fever, rash, hypotension, vomiting, and diarrhea, appear to be caused by toxins rather than by a bacteremia. Two candidates have been considered as likely mediators of the syndrome, namely pyrogenic exotoxin C (PEC) (2-4) and Staphylococcal enterotoxin $F(5)$. These now appear to be identical proteins $(6,7)$ and the enterotoxin $F$ name has been withdrawn (7). The capacity to produce PEC is a common property of $S$. aureus strains recovered from TSS patients, but the exact role of the toxin in human disease has not been established. Hitherto, PEC has been assayed by its ability to cause fever in rabbits (2-4), by its ability to induce emesis and diarrhea in monkeys (5), or by its lethality, but there is no complete animal model for TSS. Thus, there is no way of gauging the human relevance of the animal studies. For this reason and others, we have chosen to assay staphylococcal pyrogens using a human system, namely the production of interleukin-1 (IL-1) by human blood monocytes. IL-1 is a single substance, or a closely-related group of substances, that has been known by different names according to the different assay systems employed (8-11). When assayed by the production of fever, it has been known as endogenous pyrogen or leukocytic pyrogen (LP) (12) and when assayed by its ability to promote the proliferation of thymocytes, as lymphocyte-activating factor (LAF) (13). It is synthesized by and released from mononuclear phagocytes following their exposure to endotoxin, bacteria, and various substances that induce in-

1. Abbreviations used in this paper: BHI, brain heart infusion; IL-1, interleukin-1; LAF, lymphocyte-activating factor, LP, leukocytic pyrogen; MEM, minimal essential medium; PEC, pyrogenic exotoxin C; PHA, phytohemagglutinin; TH, Todd-Hewitt; TSS, toxic shock syndrome. 
flammation $(14,15)$. Its production continues long after the removal of the inducing agent. Since IL-1 production is required for the generation of fever, regardless of the nature of the exogenous pyrogenic stimulator, we anticipated that it might be involved in the fever of TSS, and we now show this to be the case. We demonstrate that TSS-associated bacteria produce material that induces IL-1 as measured in both the LP and LAF assays. The LAF assay is much the simpler method of estimating IL-1 and is now convenient to use as a routine measure of TSS toxin. Other staphylococcal products also induce IL-1 production when present in relatively high concentrations or after prior antigenic exposure. The TSS toxin, however, is so active in stimulating IL-1 production that it can be detected even when the interfering compounds have been diluted to ineffective levels.

\section{Methods}

Materials. Brain heart infusion (BHI) and Todd-Hewitt (TH) powders were obtained from Difco Laboratories, Inc., Detroit, MI. They were dissolved in pyrogen-free water and autoclaved. Phytohemagglutinin-P (PHA) was from Burroughs Wellcome Co., Research Triangle Park, NC. National reference RE-2 Escherichia coli endotoxin was kindly provided by the Bureau of Biologics, Bethesda, MD. Anti-human IL-1 was prepared and assayed as described before $(16,17)$.

Staphylococcal strains and growth. The following organisms were kindly provided by Dr. Pickrum and Dr. Stone (Proctor and Gamble Co., Cincinnati, $\mathrm{OH}$ ): strain $\mathrm{Mn}-587$ (3) which was a vaginal isolate from a TSS patient and reported by the Centers for Disease Control, Fri-1169 which was a vaginal isolate from another TSS patient (18), the Harrisburg strain which was isolated from a patient with Kawasaki's disease (2), and four strains, SA-11, SA-12, KA-1042-5VW, and SA$1052-8 \mathrm{VW}$ which were isolated from the vaginal flora of normal women. The strains designated by letters in Table I and strain 1830 were provided by Dr. Steven Schutzer. A-D were from normal women while E and $F$ were from TSS patients. Strain 1830 was isolated from a mixed infection of a wound in a male and produces Staphylococcal enterotoxin F (19). All bacteria were grown in BHI broth, and for Table I also in TH broth, aerobically with shaking, usually for $24 \mathrm{~h}$ at $37^{\circ} \mathrm{C}$. Bacteria were removed by centrifugation and the culture supernates were filtered through 0.2 $\mu \mathrm{m}$ Nalgene filters (Nalge Co., Rochester, NY).

Induction of fever in rabbits. Diluted filtrates were injected into lateral ear veins of New Zealand white female rabbits weighing 1.8-2.5 kg. Core (rectal) temperatures were monitored at intervals of $1 \mathrm{~min}$ using YSI 401 flexible thermistors (Yellow Springs Instrument Co., Yellow Springs, $\mathrm{OH}$ ) and were recorded on a DR-1A Digistrip II apparatus (Kaye Instruments, Inc., Bedford, MA). Details of the housing of rabbits and of the rabbit pyrogen test have been reported elsewhere (20).

Passive transfer of $L P$. Heparinized rabbit blood was collected from an ear artery using a 19 gauge needle. The plasma was separated and stored for $24 \mathrm{~h}$ at $4^{\circ} \mathrm{C}$. It was then injected into the ear veins of recipient rabbits who each received a bolus of $10 \mathrm{ml}$ of plasma prewarmed to $37^{\circ} \mathrm{C}$. Their rectal temperatures were recorded for $3 \mathrm{~h}$.

Production of human LP in vitro. Heparinized fresh blood from male volunteers was fractionated using Ficoll-Hypaque. The mononuclear cell layer was diluted and centrifuged at $400 \mathrm{~g}$ for $10 \mathrm{~min}$, and the cells were washed three times in isotonic saline. The cells were counted in a Coulter counter (Coulter Electronics Inc., Hialeah, FL) and adjusted to $5 \times 10^{6} \mathrm{cells} / \mathrm{ml}$ in minimal essential medium (MEM) containing 2
$\mathrm{mM}$ L-glutamine plus $100 \mathrm{U} / \mathrm{ml}$ penicillin, $100 \mu \mathrm{g} / \mathrm{ml}$ streptomycin, and $0.01 \mathrm{M}$ Hepes buffer, $\mathrm{pH}$ 7.4. A portion, $5 \mathrm{ml}$, of the monocyte suspension, in $25 \mathrm{~cm}^{2}$ plastic flasks (Falcon Plastics, Oxnard, CA), was stimulated by the addition of $100 \mu \mathrm{l}$ of a 50 -fold dilution of a filtered $S$. aureus culture filtrate and the cells were incubated at $37^{\circ} \mathrm{C}$ in a $5 \%$ $\mathrm{CO}_{2}$ incubator for $24 \mathrm{~h}$. The supernatant obtained by centrifugation at $1,000 \mathrm{~g}$ for $20 \mathrm{~min}$ was assayed by intravenous injection into rabbits whose rectal temperatures were then recorded every minute (16).

Production of LAF in vitro. A monocyte suspension, as above, was supplemented with $1 \%$ heat-inactivated male human $\mathrm{AB}$ serum and distributed in 1-ml portions to $16 \mathrm{~mm}$ diameter wells of 24-well tissue culture plate (Flow Laboratories, Inc., Hamden, CT). These were incubated at $37^{\circ} \mathrm{C}$ in $5 \% \mathrm{CO}_{2}$ for $2 \mathrm{~h}$. The supernatants were removed by aspiration and the adhering cells were washed three times with warm MEM. $S$. aureus culture filtrates of $E$. coli endotoxin diluted in MEM were added $(1 \mathrm{ml})$ to the cells. After incubation at $37^{\circ} \mathrm{C}$ for $24 \mathrm{~h}$, the monocyte culture supernatants were collected, centrifuged, and dialyzed against RPMI 1640 medium with L-glutamine (Gibco Laboratories, Grand Island, NY) at $4^{\circ} \mathrm{C}$ for $24 \mathrm{~h}$.

In one experiment, murine macrophages obtained by lavage of the peritoneal cavity were used in place of the human monocytes. They were washed three times with Hanks' balanced salt solution (HBSS), counted, suspended at $5 \times 10^{6} \mathrm{cells} / \mathrm{ml}$ in MEM, and stimulated with toxin as above.

$L A F$ assay. Murine thymi were removed from female C57 BL/6J mice and were teased apart in HBSS (15). Cells were filtered through nylon mesh, then washed three times by centrifugation $(300 \mathrm{~g}, 10 \mathrm{~min})$ in HBSS, and adjusted to $5 \times 10^{6} / \mathrm{ml}$ of RPMI containing $5 \%$ heatinactivated fetal calf serum (Hyclone Laboratory, Logan, UT), $50 \mu \mathrm{M}$ mercaptoethanol, $100 \mathrm{U} / \mathrm{ml}$ penicillin, $100 \mu \mathrm{g} / \mathrm{ml}$ streptomycin, and $0.01 \mathrm{M}$ Hepes, $\mathrm{pH}$ 7.4. $100 \mu \mathrm{l}$ portions of this cell suspension were added to each well of a 96-well microtiter plate (Nunc, Roskilde, Denmark) and incubated with PHA $(1 \mu \mathrm{g} / \mathrm{ml})$ and $100 \mu l$ of the monocyte supernate to be tested, appropriately diluted in RPMI with $5 \%$ fetal calf serum. After $48 \mathrm{~h}$ at $37^{\circ} \mathrm{C}$, every well was pulsed for a further $24 \mathrm{~h}$ with $1 \mu \mathrm{Ci}$ of tritiated thymidine $(6.7 \mathrm{Ci} / \mathrm{mmol}$; New England Nuclear, Boston, MA). DNA was collected on glass fiber filter paper using a "MASH" cell harvester (Microbiological Associates, Walkersville, MD) and counted by liquid scintillation.

\section{Results}

Demonstration of a circulating endogenous pyrogen in rabbits. When the culture supernatant of a TSS-associated strain of $S$. aureus (Fri-1 169) was injected intravenously, individual rabbits responded with a biphasic febrile response that is typical of many exogenous pyrogens (Fig. $1 \mathrm{~A}$ ). The temperature was elevated by $0.5-0.8^{\circ} \mathrm{C}$ at $1.5 \mathrm{~h}$ after injection, then fell before increasing to a major fever peak $\left(1.2-1.7^{\circ} \mathrm{C}\right)$ around 4 to $5 \mathrm{~h}$ postinjection. Other TSS-associated strains (Mn-587, 1830, Harrisburg, E and F) produced similar patterns of temperature elevation (not shown). However, the supernatant of a non-TSS strain (SA-11) did not induce a significant febrile response at any time after injection.

Plasma obtained $1.5 \mathrm{~h}$ after the injection of Fri- 169 culture filtrates was not pyrogenic when injected into other rabbits. However, plasma collected after $3 \mathrm{~h}$ produced small fevers in recipient rabbits while plasma collected after $5 \mathrm{~h}$ elevated the 

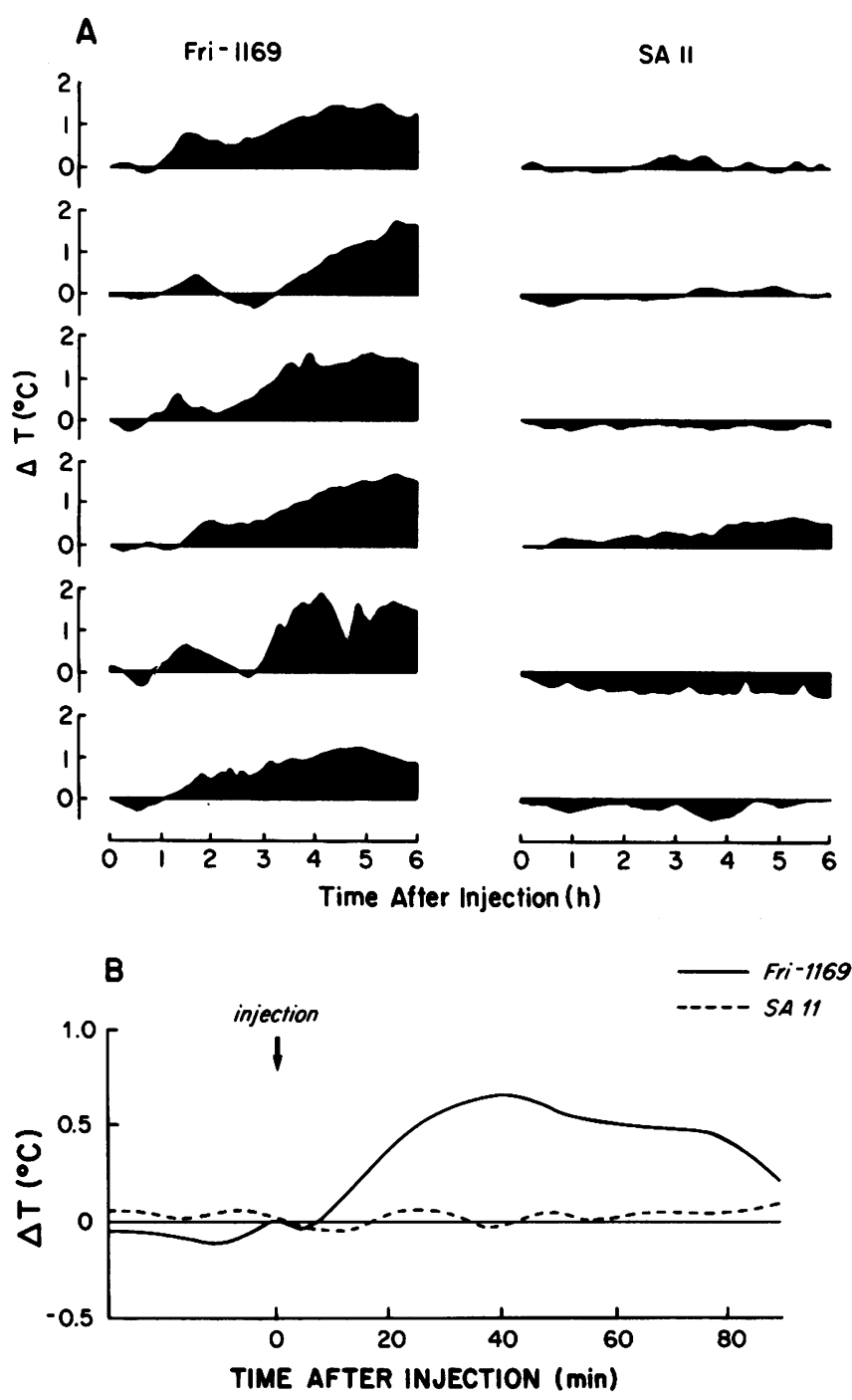

Figure $1(A)$ Individual febrile responses of rabbits injected with staphylococcal culture filtrates. Each rabbit received $1.2 \mathrm{ml}$ of MEM containing $2 \%$ of the filtrate of TSS strain Fri-1169 or control strain SA-11 grown in BHI. $(B)$ Passive transfer of circulating pyrogen. Plasma, $10 \mathrm{ml}$, obtained from rabbits in $A$ at $5 \mathrm{~h}$ was injected (arrow) into recipient rabbits. The curves each represent the mean responses of five rabbits.

recipients' body temperatures within $20 \mathrm{~min}$ and induced typical LP fevers with a mean peak of about $0.7^{\circ} \mathrm{C}$ occurring at 42 min. Plasma collected $5 \mathrm{~h}$ after injection of a filtrate of the nonTSS SA-11 filtrate did not induce any fever in recipients (Fig. $1 B)$.

Pyrogenic tolerance to TSS-associated culture filtrate. A decreased severity has been reported with subsequent bouts of TSS (21) suggesting that resistance or tolerance develops. Progressive development of tolerance is a characteristic of the human or animal response to several other exogenous pyrogens (22).
To see whether this applied to the TSS toxin, rabbits were injected daily with culture filtrates from the TSS strain Fri-1169. As shown in Fig. 2, the rabbits became somewhat less responsive to the filtrate after the third injection. After seven injections, the TSS-tolerant rabbits were challenged with human LP to test their abilities to respond to an endogenous rather than an exogenous pyrogen. They developed typical LP fevers.

Induction of human LP in vitro. Human mononuclear cells were incubated with filtrates of TSS-associated strain Fri-1169 and non-TSS strain SA-11 to test for the production of LP in vitro (Fig. 3). After $24 \mathrm{~h}$ of incubation, the supernatants were injected into rabbits. Supernates of monocytes stimulated with Fri-1169 filtrate produced typical monophasic LP fevers with peak temperature increases $40-50 \mathrm{~min}$ after injection. For comparison, Fri-1169 filtrate which had been heated to $100^{\circ} \mathrm{C}$ for 20 min was unable to stimulate LP production, as was the filtrate from a non-TSS strain (Fig. 3).

Induction of $L A F$ by $S$. aureus culture filtrates. The ability of staphylococcal filtrates to induce LAF production was investigated next. We have prepared monocytes from six male donors with entirely consistent results, but to eliminate the quantitative differences between preparations, each set of assays was performed with parallel samples of the mononuclear cells from a single donor. This allowed us to compare simultaneously the filtrates of several strains of $S$. aureus at various dilutions and with various controls. Likewise, we used a single thymocyte preparation for each experiment which allowed reliable comparisons to be made, even though the thymocyte response varies between experiments. This variation (which is commonly reported) can be seen by comparing the scales of Figs. 4-7.

A series of controls, run in parallel, is shown in Fig. $4 \mathrm{~A}$. The PHA background marked with a dashed line represents the extent of thymidine incorporation into thymocytes supplied only with monocyte culture medium. The medium from monocytes incubated alone gave a background only marginally greater than the PHA background; thus, any significant increase over this level really represents induction of IL-1. The twofold increase obtained by using a medium from monocytes incubated with BHI broth alone was about the same as that induced by $0.03-$ $0.3 \mathrm{ng} / \mathrm{ml}$ (final concentration) of endotoxin. It may represent this level of contamination of the BHI broth by endotoxin since the effect was blocked by polymyxin $B$, an antibiotic known to inhibit IL- 1 induction by endotoxins (23). We avoided any complication from endotoxin contamination in subsequent experiments (including Figs. 5-7 and Table I) by routinely including polymyxin $B$ in the monocyte culture medium. The amount of polymyxin B used had no apparent effect on the IL-1 induction by TSS toxin. None of the control strains of bacteria increased IL-1 production significantly more than the medium itself. In contrast, filtrates of the two $S$. aureus strains associated with TSS (Mn-587 and Fri-1169) dramatically increased the LAF response (Fig. 4 B). In the case of the Fri-1 169 strain, the response was still significant when the filtrate was present with the monocytes at only $0.01 \%$ ( $\mathrm{vol} / \mathrm{vol})$. 

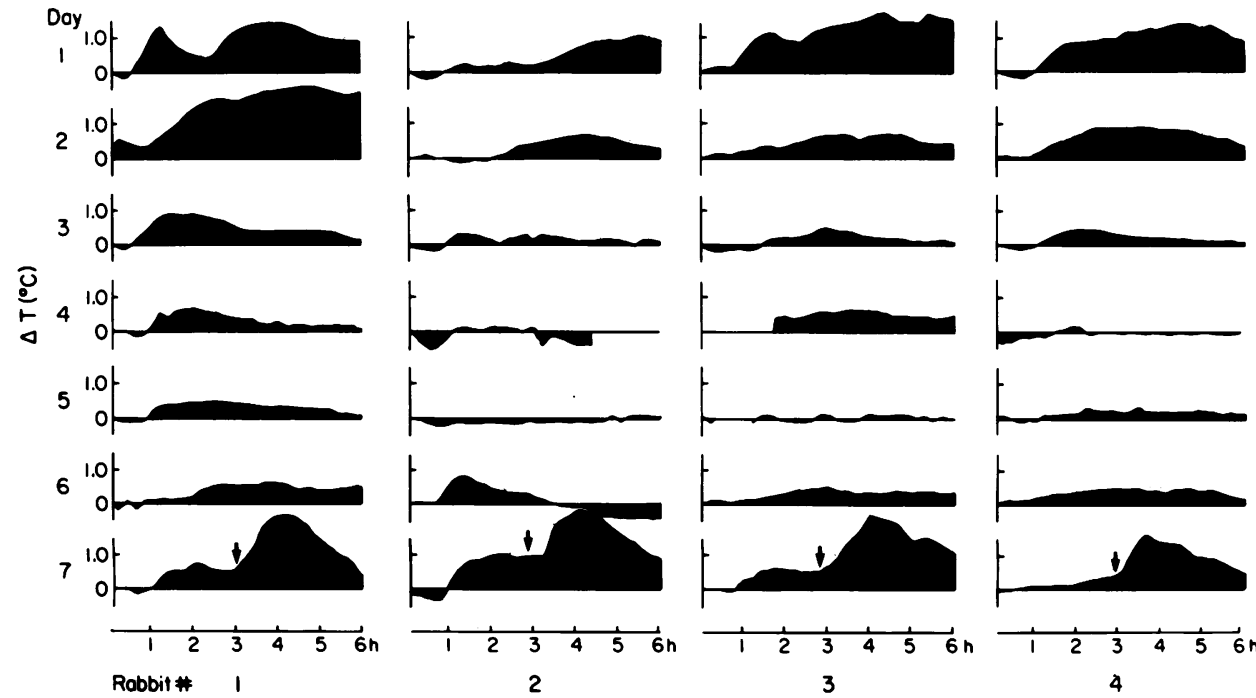

Figure 2. Development of pyrogenic tolerance to the TSS-associated toxin. Each of four rabbits was injected every day for $7 \mathrm{~d}$ with Fri1169 filtrate, as in Fig. $1 A$, and rectal temperatures were recorded. On the seventh day, the injection of bacterial filtrate was followed after 3 $h$ by the intravenous injection of 2 $\mathrm{ml}$ of a standard preparation of human IL-1 to check that the rabbits were still sensitive to IL-1. The IL-1 was prepared by incubating human monocytes with heat-killed, washed S. epidermidis.
During the LAF assay, there is a small carry-over of bacterial products from the monocyte incubation to the thymocyte culture. To control against direct effects of bacterial products upon the thymocytes, we set up incubations using bacterial filtrates that had been appropriately diluted into monocyte medium but had not been incubated with monocytes. The thymocyte cultures did display a limited response to the bacterial products themselves at concentrations of $1 \%$ (Fig. $4 \mathrm{~B}$ ). The same level of response persisted when IL-1 in the monocyte supernates was neutralized with an anti-human IL-1 serum. In both of these cases, it is most likely that the thymocytes were responding to murine IL-1 synthesized by a few macrophages present in the thymocyte preparations. Murine IL-1 is not neutralized by antihuman IL-1. In support of this explanation, we showed separately that the Fri-1 169 filtrate does indeed induce mouse macrophages to release LAF; this effect was maximal with about $0.2 \%$ filtrate while higher concentrations diminished the response (Fig. 5). The diminution may be related to our observation that high concentrations of TSS filtrates caused the macrophages to assume peculiar morphologies and to detach from the plastic surfaces of the culture dishes. On occasion, there was a similar inhibition

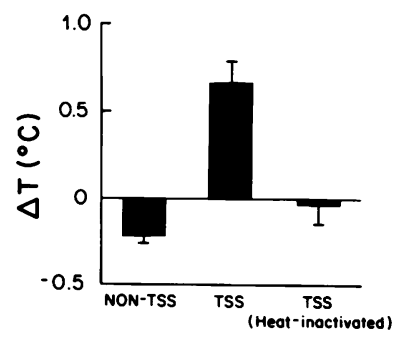

Figure 3. Production of LP in vitro. Human monocytes were stimulated with $2 \%$ filtrate of SA-11 (non-TSS), $2 \%$ filtrate of Fri-1169 (TSS), or 2\% filtrate of Fri-1169 that had been heated at $100^{\circ} \mathrm{C}$ for $20 \mathrm{~min}$ (TSS heat-inactivated). Supernates of the monocytes, $1.2 \mathrm{ml}$, were injected into three rabbits. The bars represent mean $( \pm$ SEM) peak fever which occurred within $60 \mathrm{~min}$ of the injections. of the human monocyte response by high concentrations of bacterial filtrate, but the inhibition and associated morphological changes are less evident with human monocytes than with mouse macrophages. The human monocyte response usually increases monotonically to at least $2 \%$ bacterial filtrate in the monocyte culture, as in Fig. 6.

Table I summarizes further information on these and other bacteria, making totals of five positive and eight control strains. The clear conclusion is that there is a tight correlation between TSS association of the bacteria and their ability to stimulate the production of IL-1 measured by either fever production or LAF activity. In addition, a wound-associated strain known to produce the toxin was positive in the IL- 1 assays. There are a few peculiarities in the relative LP and LAF activities; for example, the filtrates of strains $E$ and $F$ are similar in their LAFproducing abilities but are very different in the fever assay. Such peculiarities suggest complexities worthy of further study. We have found throughout that the Harrisburg strain is a relatively poor producer of the TSS toxin although it has been much used as a source of PEC.

Purified TSS toxin. We have begun to optimize the production and purification of TSS toxin by Fri-1169 bacteria by using the LAF assay as our measure. A time course of toxin production is shown in Fig. 7. Production was complete by the stationary phase and the formed toxin seemed to be stable in the culture medium during the declining phase of growth. It is convenient to use 18-24 h incubations. Both BHI and TH media are satisfactory.

Our purification procedure involves precipitation with ammonium sulfate and column chromatography on ion exchange, gel filtration, and dyematrix affinity media. At each stage, active fractions were identified by incubation with monocytes and were then pooled. Full details will be published elsewhere (Ikejima, T., C. A. Dinarello, and D. M. Gill, manuscript in preparation). As shown in Fig. $8 B$, our current product is an almost 

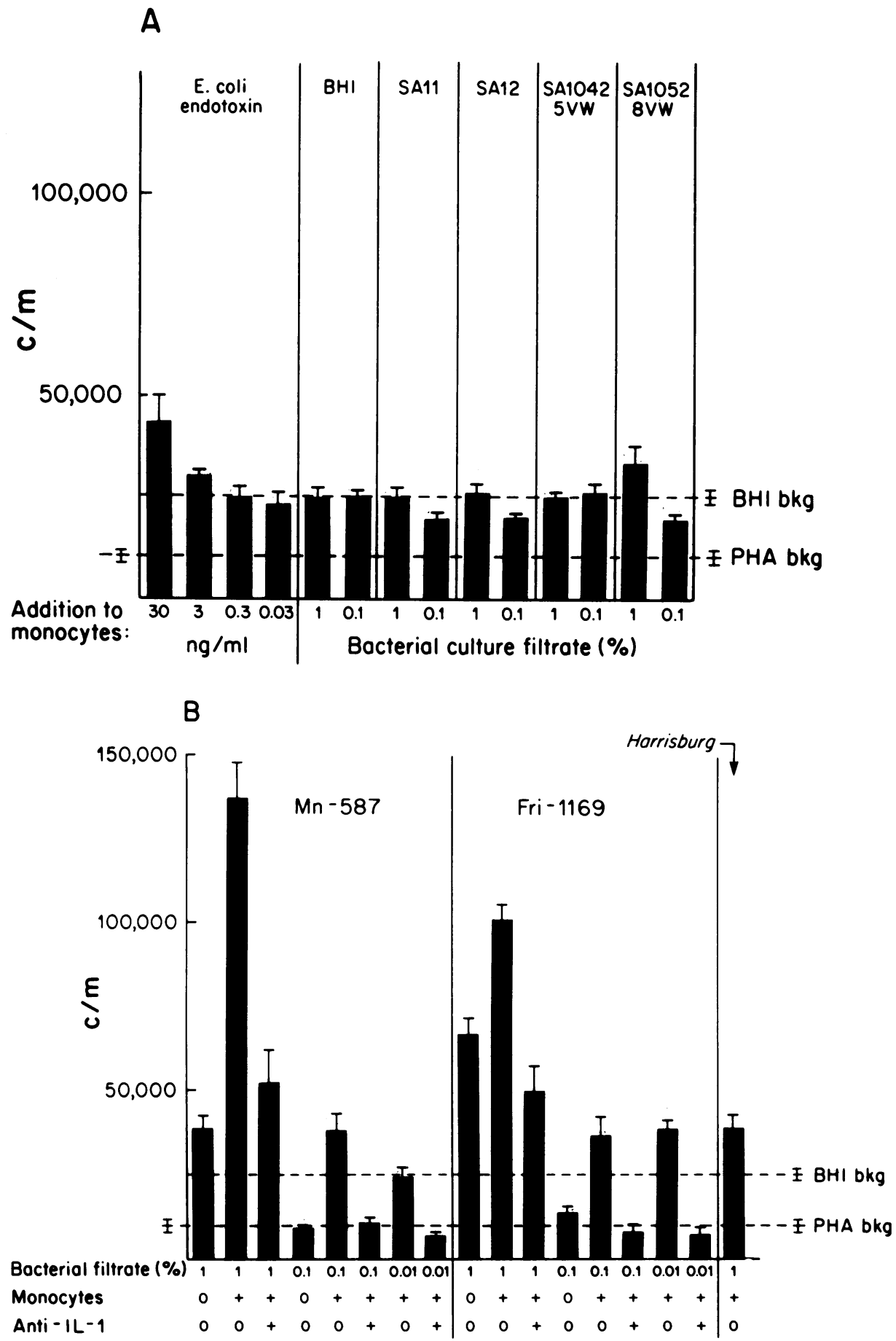

Figure 4. LAF activity of stimulated monocytes. $(A)$ Controls. Monocytes were incubated for $24 \mathrm{~h}$ with endotoxin or staphylococcal filtrates at the concentrations shown. Monocyte culture supernatants were incubated with mouse thymocytes at 1:20 dilution. The bars record the extent of subsequent thymidine incorporation into the thymocytes ( \pm SEM). PHA background (bkg), incorporation with PHA only, without monocyte supernatant; BHI background (bkg), the monocytes were incubated with BHI medium alone. $(B)$ Positive filtrates. The conditions were similar to those in $(A)$ except that, where shown, the bacterial filtrates were incubated with monocyte culture medium, without cells, and were then diluted into the thymocyte cultures. In other cases, anti-IL-1 was mixed with the monocyte supernatants at a final concentration of $1 \%$ before addition to the thymocyte cultures. $\mathrm{c} / \mathrm{m}$, counts per minute.

homogeneous protein of $M_{\mathrm{r}} \simeq 30,000$. For comparison, Fig. 8 $B$ also shows the gel pattern of the starting material. Doseresponse curves for the crude and purified materials were generated simultaneously by using the monocytes of a single donor. Significant IL-1 production was observed with as little as 0.1 $\mathrm{ng} / \mathrm{ml}$ of the purified material (Fig. $8 A$ ). As with the crude material, the purified toxin had much less effect if it was not incubated with monocytes, and as before, this apparent direct effect upon the thymocytes may be due to the stimulation of mouse macrophages among the thymocytes. 


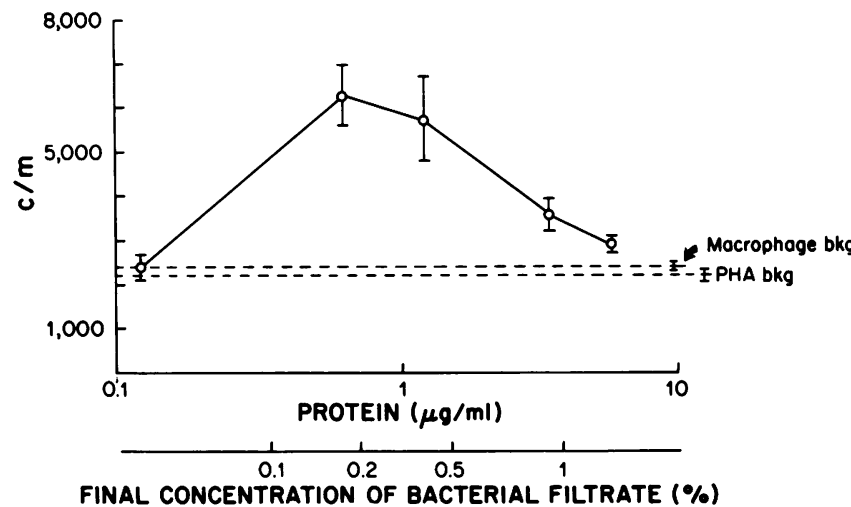

Figure 5. IL-1 production by mouse peritoneal macrophages stimulated with Fri-1 169 filtrate, at the concentrations shown, in the presence of $5 \mu \mathrm{g} / \mathrm{ml}$ polymyxin B. Thymocytes were incubated with macrophage supernatant at 1:20 dilution. bkg, background; $\mathrm{c} / \mathrm{m}$, counts per minute.

\section{Discussion}

The experiments described show that two strains of $S$. aureus isolated from patients with TSS produce a toxin that induces the synthesis and release of IL-1. IL-1 production in vivo was demonstrated by the presence of endogenous pyrogen in the circulation of rabbits that had been injected with culture filtrates of TSS bacteria. Similarly, production of IL-1 in vitro was shown

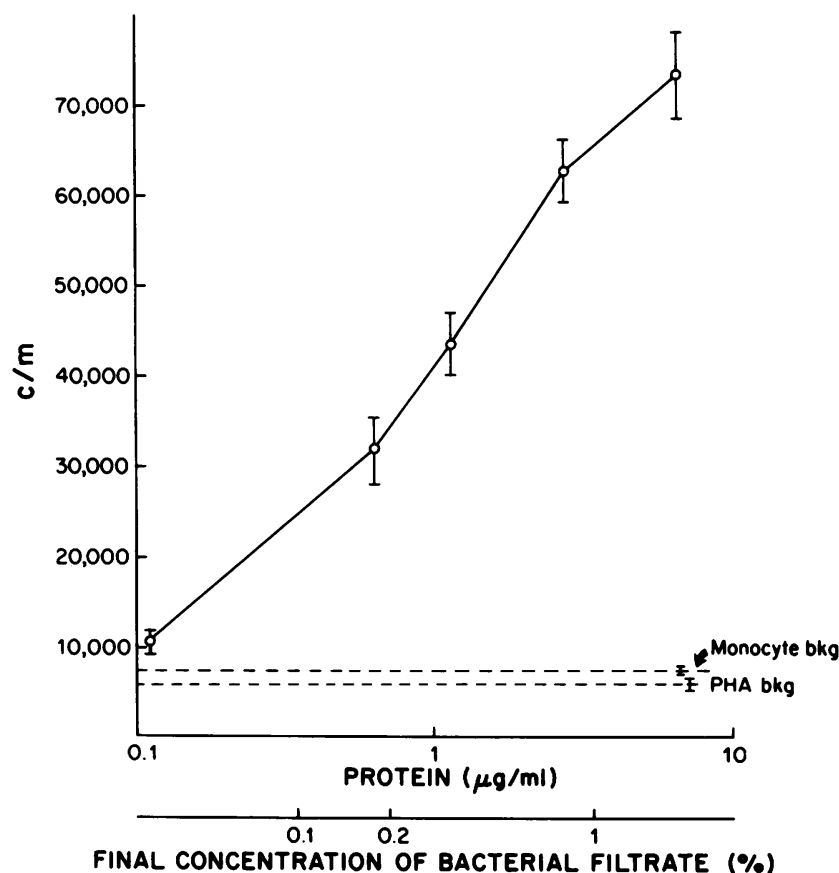

Figure 6. IL-1 production by human monocytes stimulated with Fri1169 filtrate, as in Fig. 5. bkg, background; c/m, counts per minute. by: ( $a$ ) measuring the production of LP by human mononuclear cells incubated with filtrates, and $(b)$ by measuring the production of LAF from both adherent human monocytes and murine peritoneal macrophages incubated with filtrates. Anti-human IL-1 was used to confirm that the material produced by the monocytes was in fact IL-1. To our knowledge, this is the first demonstration that human IL-1 is induced by material derived from TSS-associated $S$. aureus. Hitherto, lethality for monkeys and rabbits and pyrogenicity for rabbits have been the measures of the biological activity of TSS bacterial products. Obviously, these are not the most relevant assays. In particular, they may fail to reveal any unique sensitivity of human cells for the toxin, which seems a reasonable possibility given the marked febrile and inflammatory nature of the disease.

The demonstration of IL-1 production may be important in explaining the pathogenesis of TSS. IL-1 is presently considered to be either a single polypeptide or a family of closelyrelated polypeptides which mediates several components of the acute-phase response to infectious, inflammatory, and immunological diseases $(10-12,14,15)$. IL-1 was originally identified as endogenous or leukocytic pyrogen but the properties of LP have recently been found to be indistinguishable from those of leukocytic endogenous mediator and LAF. Thus, it is now probable that a variety of activities of stimulated phagocytes are all properties of the same molecule or of a set of closely related molecules. Among these activities are the modulation of lymphokine production and lymphocytic reactivity attributed to LAF, and neutrophilia, hypo-ferremia, and acute phase protein synthesis, which are attributed to leukocytic endogenous mediator (15). Thus, the formation and effects of IL-1 may explain much of the pathogenesis of TSS. Moreover, it seems that IL1's actions on various cells may all be mediated by an increase in arachidonic acid. Such an increase is followed by increases in the amounts of prostaglandins and leukotrienes (24), both highly active substances that are likely to contribute to other aspects of the syndrome, such as diarrhea and hypotension (25).

The potency of the toxin may contribute further to our understanding of the pathogenesis of TSS. The pyrogen appears to be a protein and may be related, if not identical, to the toxin described by others (2-7). The material which we have purified is effective at as little as $0.1 \mathrm{ng} / \mathrm{ml}$ and represents most of the activity in the crude filtrate. It is thus more potent than endotoxin.

In our initial experiment, we found that unused bacterial growth medium induced a small but real IL-1 production that was sensitive to polymyxin B and was therefore most probably due to contaminating endotoxin. Such contamination was not surprising since endotoxins are ubiquitous and are not readily destroyed by autoclaving (26). In subsequent experiments, we included in the monocyte medium polymyxin $\mathrm{B}$, a cationic antibiotic which prevents endotoxins from inducing IL-1 both in vivo and in vitro $(23,27)$. Since polymyxin B did not affect the ability of the culture filtrates from TSS strains to induce IL-1, it is clear that the biological activity of TSS-derived material 
Table I.

\begin{tabular}{|c|c|c|c|c|c|c|}
\hline \multirow{3}{*}{$\begin{array}{l}\text { Culture medium: } \\
\text { Filtrate concentration: }\end{array}$} & \multicolumn{2}{|l|}{ Todd-Hewitt } & \multicolumn{4}{|c|}{ Brain-heart } \\
\hline & \multicolumn{2}{|c|}{ Peak febrile response $(\mu l / k g)^{*}$} & \multicolumn{4}{|c|}{ LAF activity $(\%) \ddagger$} \\
\hline & 36 & 12 & 0.1 & 0.02 & 0.1 & 0.02 \\
\hline Medium & $0.0 ; 0.0 ; 0.0$ & & 3 & & 3 & \\
\hline \multicolumn{7}{|l|}{ Control strains } \\
\hline A & - & $0.1 ; 0.1 ; 0.1$ & 3 & 3 & $3 \pm 1$ & $4 \pm 1$ \\
\hline B & - & $0.4 ; 0.0 ; 0.0$ & 3 & 2 & 5 & $5 \pm 1$ \\
\hline C & - & $0.1 ; 0.0 ; 0.0$ & 2 & 2 & $4 \pm 1$ & 3 \\
\hline D & $0.0 ; 0.0 ; 0.2$ & $0.0 ; 0.0 ; 0.0$ & 2 & 2 & $5 \pm 1$ & $4 \pm 1$ \\
\hline \multicolumn{7}{|c|}{ TSS-associated strains } \\
\hline Fri-1169 & - & $0.7 ; 1.6 ; 1.4$ & $47 \pm 6$ & $24 \pm 4$ & $56 \pm 3$ & $39 \pm 2$ \\
\hline Mn-587 & $1.1 ; 0.7 ; 0.0 ; 1.3 ; 0.3$ & $0.1 ; 0.1 ; 0.1$ & $19 \pm 2$ & $4 \pm 2$ & $28 \pm 2$ & $12 \pm 1$ \\
\hline Harrisburg & - & $0.5 ; 0.7 ; 0.8$ & $26 \pm 4$ & $24 \pm 2$ & $38 \pm 4$ & $26 \pm 4$ \\
\hline E & - & $2.4 ; 1.5 ; 1.8$ & $36 \pm 5$ & $21 \pm 4$ & $45 \pm 5$ & $31 \pm 2$ \\
\hline $\mathbf{F}$ & - & $0 ; 0.3 ; 0.4$ & $35 \pm 5$ & $17 \pm 2$ & $32 \pm 1$ & $32 \pm 2$ \\
\hline \multicolumn{7}{|c|}{ Wound-associated strain } \\
\hline 1830 & - & $1.4 ; 1.1 ; 1.9$ & $46 \pm 3$ & $46 \pm 3$ & $65 \pm 4$ & $53 \pm 4$ \\
\hline
\end{tabular}

* Peak fevers, degrees Celius, or individual rabbits. $¥$ Tritiated thymidine incorporated into thymocytes incubated with medium of monocytes (1:20 dilution) that had been exposed to filtrates at the concentrations shown. Data are expressed as mean counts per minutes $\times 10^{-3}$ ( \pm SD) of triplicate samples. SD $<1$ are omitted.

can be separated from that of endotoxin. This is an important point since endotoxins have been the most potent known pyrogens and stimulators of the acute phase responses in humans $(28,29)$.

Filtrates from TSS-strains induced biphasic fevers in rabbits similar to those induced by some other exogenous pyrogens (22). The major fever that occurred after several hours was prolonged and endogenous pyrogen was shown to be present

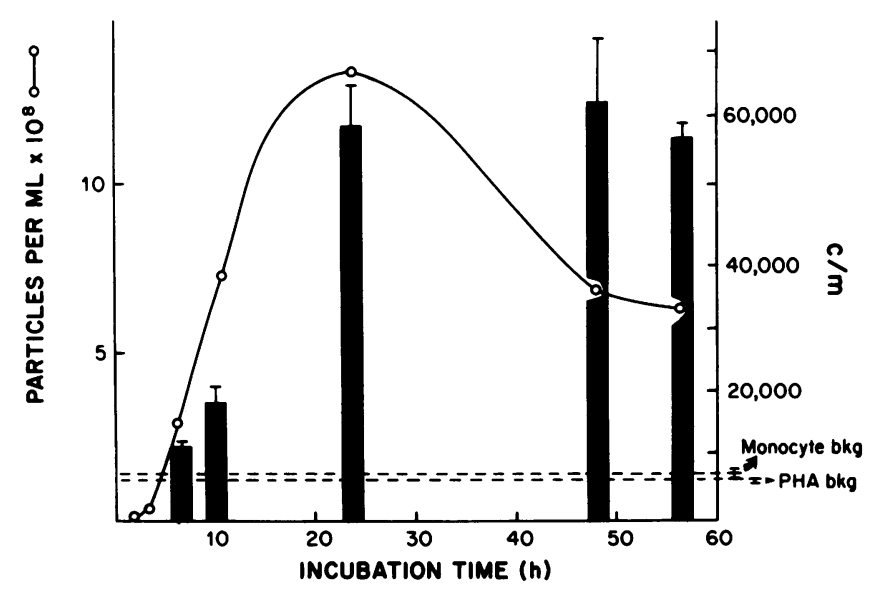

Figure 7. Toxin production and growth of Fri-1169 bacteria. IL-1 was estimated as in Fig. 5. Particle counts were calculated as $10^{8}$ $\times \mathrm{OD}_{650}$. bkg, background; $\mathrm{c} / \mathrm{m}$, counts per minute. at its peak. In addition, there was an earlier febrile peak, 1.5$2 \mathrm{~h}$ after toxin injection, that was apparently not associated with circulating endogenous pyrogen and may represent a direct effect of the toxin on the thermoregulatory center. Since a plasma taken after 1.5-2 $\mathrm{h}$ produced no febrile response in recipient rabbits, it seems likely that the injected toxin had already been cleared from the circulation. Other exogenous pyrogens are also rapidly cleared following intravenous injection. They can be found then in the cells of the reticuloendothelial system (22), which is not surprising since these are major sources of IL-1.

Daily injections of culture filtrates from TSS strain Fri-1 169 induced pyrogenic tolerance, although the recipient rabbits remained responsive to human IL-1. Such pyrogenic tolerance has been observed with other exogenous pyrogens (22).

Finally, certain aspects of the LAF assay merit attention. High concentrations of the bacterial filtrate had some effect when they were added directly to the LAF assay system. We considered whether this response could represent a direct effect of the toxin upon thymocytes, as others have suggested $(3,30)$. This may indeed occur but the major effect seems more likely to be due to the induction of murine IL-1 from a few macrophages that contaminate the thymocyte preparation. Lower concentrations of the bacterial filtrates induce human monocytes to produce IL-1 but have no effect when carried over into the thymocyte culture. The bona fide human IL-1 then produced is completely neutralized by anti-human IL-1.

It is relevant to inquire whether any toxin-lymphocyte interactions might occur. While we cannot rule out this possibility, 


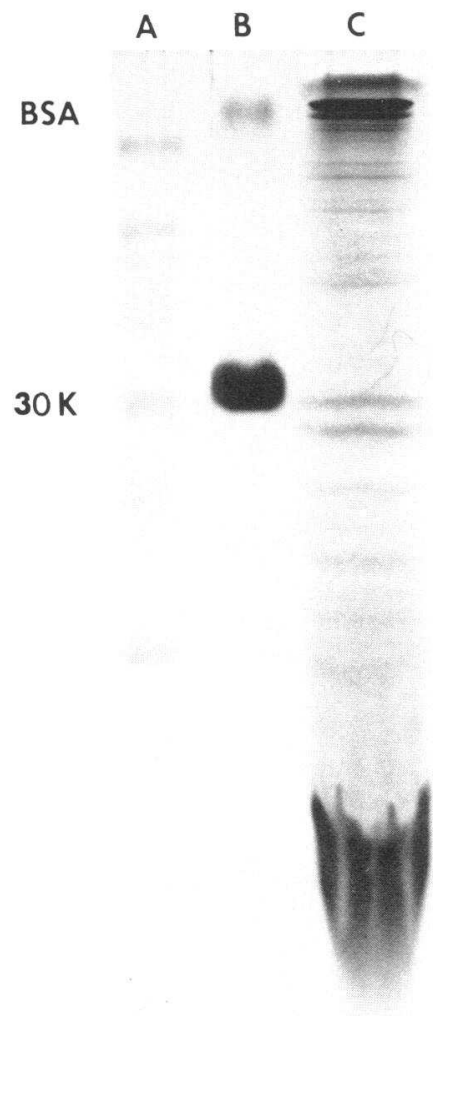

\section{A}

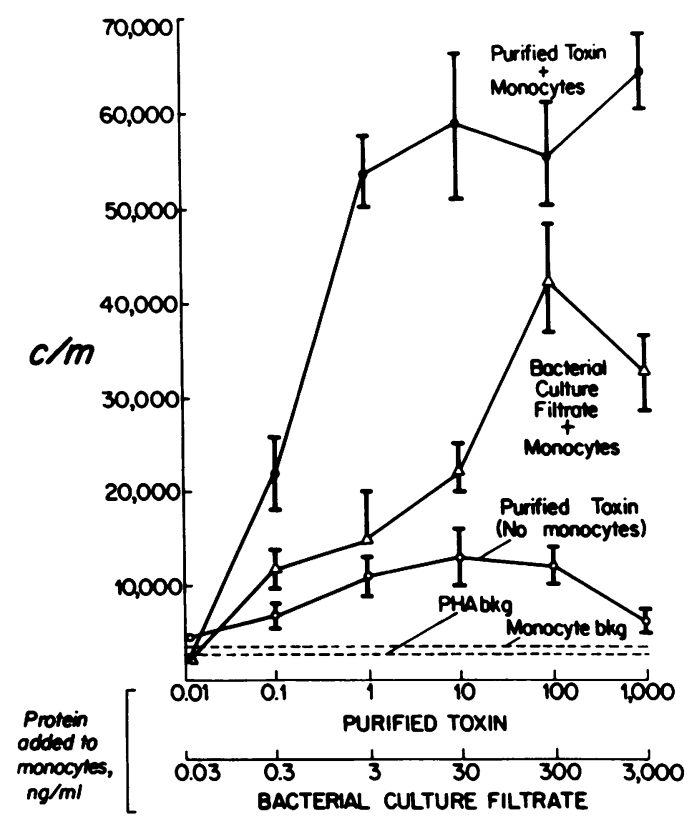

Figure 8. $(A)$ Comparison of the induction of IL-1 by crude and purified toxin. Protein was added to the monocyte cultures at the final concentrations shown. The original culture filtrate contained $300 \mu \mathrm{g}$ of protein $/ \mathrm{ml}$. In the case indicated, the purified toxin was diluted in the absence of monocytes and later added to mouse thymocytes. bkg, background; $c / m$, counts per minute. $(B)$ An analysis of the crude

such interactions are unlikely to affect the assays significantly because there are few remaining lymphocytes in the monocyte preparations. Thus, the combined monocyte-thymocyte assay does appear to measure IL-1 production from toxin-stimulated monocytes.

In previous studies on PEC, the minimum pyrogenic dose was defined as the amount per kilogram of rabbit that would induce a rise in temperature of $0.5^{\circ} \mathrm{C}$ at $4 \mathrm{~h}(2,3,30)$. Several extensive investigations have shown that this is not an accurate measure (31-36). For example, individual rabbits vary considerably in their responses; other staphylococcal products (peptidoglycan and teichoic acids) cause fever in rabbits, and rabbits may develop pyrogenic tolerance to these staphylococcal products. One is limited in the number of samples that can be assayed at one time. The LAF assay of IL-1, in contrast, allows us to test many samples of TSS-derived materials at once, to assay and purified materials by SDS-polyacrylamide gel electrophoresis. The band marked BSA in the purified toxin track is a trace of the bovine serum albumin that we had added as a protective agent during the later stages of toxin purification. It has no LAF-inducing activity.

them in a quantitative manner, to compare them directly with other toxins and activators of IL-1 production, and in the future, to determine the effects of drugs and antibodies on the monocyte response.

\section{Acknowledgments}

The authors wish to thank Gail LoPreste, Lorraine Barrie, Harvey M. Pickrum, Roger L. Stone, and James S. Widder for their help and advice.

This work was supported by funds from the Procter and Gamble Company, Cincinnati, $\mathrm{OH}$ and by National Institutes of Health grants AI-15614 and AI-16928.

\section{References}

1. Todd, J., M. Fishant, and F. Kapral. 1978. Toxic-shock syndrome associated with phage-group 1 Staphylococci. Lancet. II:1116-1118. 
2. Schlievert, P. M., D. J. Schoettle, and D. W. Watson. 1979. Purification and physicochemical and biological characterization of a staphylococcal pyrogenic exotoxin. Infect. Immun. 23:609-617.

3. Schlievert, P. M., K. N. Shands, B. B. Dan, G. P. Schmid, and R. D. Nishimura. 1981. Identification and characterization of an exotoxin from Staphylococcus aureus associated with toxic shock syndrome. J. Infect. Dis. 143:509-516.

4. Schlievert, P. M., M. T. Osterholm, J. A. Kelly, and R. D. Nishimura. 1982. Toxin and enzyme characterization of Staphylococcus aureus isolates from patients with and without toxic shock syndrome. Ann. Intern. Med. 96:937-939.

5. Bergdoll, M. S., B. A. Crass, R. F. Reiser, R. N. Robbins, and J. P. Davis. 1981. A new staphylococcal enterotoxin, enterotoxin F, associated with toxic-shock syndrome Staphylococcus aureus isolates. Lancet. I:1017-1021.

6. Bonventre, P. F., L. Weckbath, J. Staneck, P. M. Schlievert, and M. Thompson. 1983. Production of Staphylococcal enterotoxin F and Pyrogenic exotoxin C by Staphylococcus aureus isolates from Toxic Shock Syndrome-associated sources. Infect. Immun. 40:1023-1029.

7. Reiser, R. F., R. N. Robbins, G. P. Khoe, and M. S. Bergdoll. 1983. Purification and some physical properties of toxic-shock toxin. Biochemistry. 22:3907-3912.

8. Rosenwasser, L. J., C. A. Dinarello, and A. S. Rosenthal. 1979. Adherent cell function in murine T-lymphocyte antigen recognition. IV. Enhancement of murine T-cell antigen recognition by human leukocytic pyrogen. J. Exp. Med. 150:709-714.

9. Murphy, P. A., P. L. Simon, and W. F. Willoughby. 1980. Endogenous pyrogens made by rabbit peritoneal exudate cells are identical with lymphocyte-activating factors made by rabbit alveolar macrophages. J. Immunol. 124:2498-2501.

10. McAdam, K. P. W. J., and C. A. Dinarello. 1980. Induction of serum amyloid A synthesis by human leukocytic pyrogen. In Bacterial Endotoxins and Host Response. M. D. Agawal, editor. Elsevier/North Holland, Amsterdam. 167-178.

11. Sztein, M. D., S. N. Vogel, J. D. Sipe, P. A. Murphy, S. B. Mizel, J. J. Oppenheim, and D. L. Rosentstreich. 1981. The role of macrophages in the acute-phase response: SAA inducer is closely related to lymphocyte activating factor and endogenous pyrogen. Cell. Immunol. 63:164-176.

12. Dinarello, C. A., and S. M. Wolff. 1982. Molecular basis of fever in humans. Am. J. Med. 72:799-819.

13. Gery, I., R. K. Gershon, and B. H. Waksman. 1972. Potentiation of the T-lymphocyte response to mitogens. I. The responding cell. $J$. Exp. Med. 136:128-142.

14. Dinarello, C. A. 1984. Interleukin-1. Rev. Infect. Dis. 6:51-95.

15. Kampschmidt, R. F. 1981. Leukocytic endogenous mediator/ endogenous pyrogen. In The Physiologic and Metabolic Responses of the Host. M. D. Powanda and P. G. Canonico, editors. Elsevier/North Holland, Amsterdam. 55-74.

16. Dinarello, C. A., L. Renfer, and S. M. Wolff. 1977. The production of antibody against human leukocytic pyrogen. J. Clin. Invest. 60:465472.

17. Rosenwasser, L. J., and C. A. Dinarello. 1981. Ability of human leukocytic pyrogen to enhance phytohemagglutinin induced murine thymocyte proliferation. Cell. Immunol. 163:134-142.

18. Kreiswirth, B. N., R. P. Novick, P. M. Schlievert, and M. Bergdoll.
1982. Genetic studies on staphylococcal strains from patients with toxic shock syndrome. Ann. Intern. Med. 96:974-977.

19. Kreiswirth, B. N., S. Löfdahl, M. J. Betley, M. O'Reilly, P. M. Schlievert, M. S. Bergdoll, and R. P. Novick. 1983. The toxic shock syndrome exotoxin structural gene is not detectably transmitted by a prophage. Nature (Lond.). 305:709-712.

20. Wolff, S. M., J. H. Mulholland, and S. B. Ward. 1965. Quantitative aspects of the pyrogenic responses of rabbits to endotoxin. J. Lab. Clin. Med. 65:268-276.

21. Davis, J. P., M. T. Osterholm, C. M. Helms, J. M. Vergeront, L. A. Wintermeyer, J. C. Forfang, L. A. Judy, J. Rondeau, W. L. Schell, and The Investigation Team. 1982. Tri-state toxic shock study: evaluation of case definition and prevention of recurrence. Ann. Intern. Med. 96:903905.

22. Dinarello, C. A., and S. M. Wolff. 1982. Exogenous pyrogens. In Pyretics and Antipyretics. A. S. Milton, editor. Springer Verlag, Berlin. 73-112.

23. Duff, G. W., and E. Atkins. 1982. The inhibitory effect of polymyxin B on endotoxin-induced endogenous pyrogen production. J. Immunol. Methods. 52:333-340.

24. Dinarello, C. A., S. O. Marnoy, and L. J. Rosenwasser. 1983. Role of arachidonate metabolism in the immunoregulatory function of human leukocytic pyrogen/lymphocyte-activating factor/interleukin-1. J. Immunol. 130:890-895.

25. Samuelsson, B. 1983. Leukotrienes: mediators of immediate hypersensitivity reactions and inflammation. Science (Wash. DC). 220:568-575.

26. Elin, R. J., and S. M. Wolff. 1982. Bacterial endotoxins. In CRC Handbook on Microbiology. A. I. Laskin and H. A. Lechevalier, editors. CRC Press, Inc., Cleveland. 4:253-272.

27. van Miert, A. S., and C. T. van Duin. 1978. Further studies on the antipyretic action of polymyxin B in pyrogen-induced fever. Arzneim. Forsch. 28:2246-2251.

28. Wolff, S. M. 1973. Biological effects of bacterial endotoxins in man. J. Infect. Dis. 128(Suppl.):251-256.

29. Elin, R. J., L. Chedid, S. M. Wolff, K. P. W. J. McAdam, F. Audibert, C. Bernard, and F. Oberling. 1981. Properties of the reference endotoxin and its phthalylated derivative in humans. J. Infect. Dis. 144:329-338.

30. Schlievert, P. M., and J. A. Kelly. 1982. Staphylococcal pyrogenic exotoxin type C: further characterization. Ann. Intern. Med. 96:982985.

31. Atkins, E., and L. R. Freedman. 1963. Studies in staphylococcal fever. I. Responses to bacterial cells. Yale J. Biol. Med. 35:451-471.

32. Atkins, E. 1963. Studies in staphylococcal fever. II. Responses to culture filtrate. Yale J. Biol. Med. 35:472-488.

33. Atkins, E. 1963. Studies in staphylococcal fever. III. Tolerance to culture filtrates. Yale J. Biol. Med. 35:489-501.

34. Bodel, P. T., and E. Atkins. 1964. Studies in staphylococcal fever. IV. Hypersensitivity to culture filtrates. Yale J. Biol. Med. 37:130144.

35. Bodel, P. T., and E. Atkins. 1965. Studies in staphylococcal fever. V. Staphylococcal filtrate pyrogen. Yale J. Biol. Med. 38:282298.

36. Atkins, E., and S. I. Morse. 1967. Studies in staphylococcal fever. VI. Responses induced by cell walls and various fractions of Staphylococci and their products. Yale J. Biol. Med. 39:297-311. 